\title{
Prevalence of Antenatal Depressive Symptoms and Associated Factors among Pregnant Women in Maichew, North Ethiopia: An Institution Based Study
}

\author{
Tilahun Belete Mossie $^{1 *}$, Adam Kiros Sibhatu ${ }^{1}$, Abate Dargie ${ }^{2}$, \\ Ashenafi Damte Ayele ${ }^{1}$
}

OPEN ACCESS

Citation: Tilahun Belete Mossie, Adam Kiros Sibhatu, Abate Dargie, Ashenafi Damte Ayele. Prevalence of Antenatal Depressive Symptoms and Associated Factors among Pregnant Women in Maichew, North Ethiopia: An Institution Based Study. Ethiop J Health Sci 2017;27(1):59-66.

http://dx.doi.org/10.4314/ejhs.v27i1.8

Received: October 122016

Accepted: November 14, 2016

Published: January 1, 2017

Copyright: Tilahun BM, et al. This is an

open access article distributed under the

terms of the Creative Commons

Attribution License, which permits unrestricted use, distribution, and reproduction in any medium, provided the original author and source are credited.

Funding: None

Competing Interests: The authors

declare that this manuscript was approved

by all authors in its form and that no

competing interest exists

Affiliation and Correspondence:

${ }^{1}$ Psychiatry Unit, School of Nursing,

College of Health Sciences, Mekelle

University, Ethiopia

${ }^{2}$ College of Health Sciences, Debre Birhan University, Debre Birhan, Ethiopia

"Email: tilahunbe100@gmail.com

\section{ABSTRACT}

BACKGROUND: Antenatal depression is one of the common problems during pregnancy with a magnitude of $20 \%$ to $30 \%$ globally. It can negatively endanger women's and off springs lives. As there are scarce reports on this area in Northern Ethiopia, it is important to carry out different studies that explore the magnitude of the problem and related factors in rural areas. The aim of this study is thus to assess the magnitude of antenatal depressive symptoms and associated factors among women at Maichew Town, North Ethiopia.

METHODS: A facility based cross sectional study was conducted among 196 pregnant women from April to June 2015. Pregnant women who had antenatal care follow-ups at the public health facilities were included in the study. Through proportional allocation to each facility, systematic random sampling technique was used to select the study participants. We used the local language version of Beck Depression Inventory to assess depressive symptoms with a cutoff point of 14 or more. Data was collected by trained Psychiatric Nurses; data entry and analysis were processed by SPSS window 20. The level of significance was determined using odds ratio and $95 \%$ confidence interval.

RESULT: About $16.3 \%$ of the participants had never given birth before, and $46.4 \%$ and $42.3 \%$ were in the third and second trimesters of pregnancy respectively. Unwanted pregnancy was reported by $25.5 \%$ of the participants. Among those with previous pregnancy, $7.1 \%$ had previous obstetric complication. The magnitude of depression was 31.1\%. Pregnant women with low level of income $(A O R=3.66(95 \% C I ; 1.12,11.96))$, unmarried $(A O R=4.07(95 \%$ CI; 1.18, 14.04) $)$ and house wives $(A O R=4.24$ $(1.38,13.03))$ were risk groups for depression.

CONCLUSION: Antenatal depression is a common problem; thus screening activities of depression in antenatal care services should be emphasized with more concern to unmarried women, those with low level of income and house wives.

KEYWORDS: Antenatal depression, Mental Distress, Depression, Depression during pregnancy 


\section{INTRODUCTION}

One of the significant components of reproductive health but with less attention is mental health. At least one in ten women in developed nations and two in five women in the developing world develop depression during pregnancy $(1,2)$.

Antenatal depression is characterized by sad mood, loss of interest, hopelessness, decreased energy, difficulty to think, poor concentration, disturbed sleep and trouble with appetite along with inability to feel happiness $(3,4)$. Sometimes, death wish and suicidal thoughts or attempts may also occur $(3,4)$.

Uterine irritability, pregnancy induced hypertension, pre-eclampsia, postpartum bleeding, pre-term delivery and also emotional and behavioral problems (in the offspring) are common effects of antenatal depression (3,5-10). It can also present with poor self-care and poor medical (antenatal care) follow-up (5-10). The probability of using different psycho-active substances by the mother is increased and has a potential effect on the fetus (3,5-10). In addition, decreased head circumference, low birth weight and decreased reflex response are common effects on the fetus (11-14). Contrary to this, a study from Pakistan concluded that antenatal depression is not associated with low birth weight (15). Post partum depression (depression after giving birth) was strongly associated with antenatal depression (16 - 20). Similarly, it exposes the offspring to childhood maltreatment and at risk of developing mental health problems in the future (21).

A number of factors can predispose to antenatal depression. Of these, some are poor ANC follow up, malnutrition, facing with a stressor, past history of mental illness, pregnancy related complications and previous operative delivery (22).

Antenatal depression among low income Brazilian women indicated that the prevalence of depression in the third trimester of pregnancy was $38 \%$. Also, depression in the first six months after delivery was reported among $43 \%$ of the women (23). Diverse Chinese based studies reported that the magnitude of antenatal depression lies from at least $5.5 \%$ to a maximum of $23.1 \%(24,25)$. Similarly the prevalence of depression in the second and third trimesters of pregnancy was $18.9 \%$ and $22.1 \%$ respectively in Hong Kong (26). In Taiwan it was $20 \%$ during the third trimester (27).

A study from Mumbai reported that the prevalence of antenatal depression was $9.2 \%$; and it was related with unplanned pregnancy, history of abortion and obstetric complications (28). In South Africa, similar prior studies reported $21 \%$ $47 \%$ magnitude of antenatal depression that was predicted by single marital status, low socio economic status, recent stressful life events, unplanned pregnancy, childhood trauma and pastyear intimate partner violence $(29,30)$.

In Ethiopia, the magnitude of antenatal depression is found to be in a range of $24.9 \%$ $30 \%$ in Addis Ababa; $23 \%$ at Gondar and 25.6\% at Shashemene $(31,32,33,34,35)$. In addition, the independent factors associated include obstetric problems, unplanned pregnancy, past mental illness, unmarried by marital state, lack of family support, lack of spouse support, violence during pregnancy, low socio economic status, younger age, first pregnancy and poor antenatal care follow up $(31,32,33,34,35)$.

In general, antenatal depression has enormous effects on the mother and her offspring, and it can complicate the pregnancy so that different research activities that try to explain the problem are important. This study provides relevant information about the magnitude of the problem and related factors at Maichew Town where there has been no prior similar study. This is crucial to the public health facilities, regional health bureau and other stakeholders. Because it provides relevant information that can be used as an input in planning different packages to address comprehensive need of pregnant women in rural areas, the main objective of this study is to determine the magnitude of antenatal depression and to identify the independent related factors among pregnant women at Maichew, Tigray, Ethiopia.

\section{METHODS AND SUBJECTS}

Setting: The study was conducted at Maichew Town, which is found in the northern part of Ethiopia in Tigray region. Maichew is a town located at $665 \mathrm{~km}$ north of Addis Ababa. Its

DOI: http://dx.doi.org/10.4314/ejhs.v27i1.8 
altitude is 2479 meters above sea level. According to Ethiopia's agro-ecological setting, Maichew and its environments are classified under the Weinadega (semi-temperate zone) (36).

Based on the 2007 national census conducted by the Central Statistical Agency of Ethiopia (CSA), this town has a total population of 23,419 , of whom 11,024 are men and 12,395 women. About $95.3 \%$ of the population is Orthodox Christian. Maichew has one hospital and two health centers which they have antenatal care follow-up services (36).

Design: Facility based quantitative cross sectional study was conducted. All women who had ANC follow-up at public health facilities were the source population whereas those mothers who came to ANC follow-up during the study period and who were randomly selected were the population studied. Pregnant women with serious medical illness at follow-up or those who had known depressive disorder were excluded from the study.

Sampling and sampling procedure: The sample size was determined using the following assumptions: $95 \%$ level of confidence interval, a $5 \%$ margin of error and a proportion of $30 \%$ (magnitude of antenatal depression at Addis Ababa $(31,32,33)$ and a $10 \%$ non-response rate. Based on this assumption, the actual sample size for the study was 322 . Since the source population was 600 , we employed correction formula, and the final sample size became 209.

All public health facilities (two health centers and one hospital) in the town were included in the study. After reviewing a one-month follow-up record of the facilities, proportional allocation was considered, and participants were selected through systematic random sampling technique. To determine the degree of association, sociodemographic factors, gynecological and obstetric conditions and health related factors were taken as independent factors.

Data collection and quality assurance: The study was conducted from April to June 2015. Local language version of interviewer administered structured questionnaire was used to collect data. Beck Depression Inventory tool was also used to assess depressive symptoms. Data was collected by trained psychiatric nurses after a trail of pre-test in $5 \%$ of the samples at private clinics. Each filled questionnaire was checked daily for completeness and accuracy.

To assure quality, data collectors were trained. In addition, the questionnaire was translated from English to a local language (Tigrigna) and back to English by professional translators with the assistance of a mental health specialist. Also, pre-test was carried out in 12 mothers who had follow-ups at private clinics. Supervision was employed throughout the data collection period. Besides, daily check of the data for completeness and consistency was under taken.

Operational definition: According to Beck's Depression Inventory, a score of 14 or more was considered having depressive symptoms. Also, subtypes of depression were interpreted as a score of 0-13 (minimal depression), 14-19 (mild depression), 20-28 (moderate depression) and 2963 (severe depression).

Ethical clearance: Ethical clearance and permission were was obtained from the Ethical Review Committee of the College of Health Science, Mekelle University and the respective health institutions respectively before the data collection process started. All the study participants were informed about the objectives and importance of the study. In addition, brief information on the importance of early identification and proper treatment of antenatal depression were discussed. Also, the study subjects were informed that they could skip question or questions that they did not want to answer fully or partly. They were also told to stop the interviewing process at any time if they wanted to do so. Participants with moderate and above levels of depression were referred to the nearby psychiatry clinic for further evaluation.

\section{RESULTS}

A total of 196 mothers participated in the study with a response rate of $93.8 \%$. The large portion (92.3\% ( 181)) of pregnant mothers were married; $34.7 \%$ ( 68 mothers) were in the age group between 26-30 years. About 73(37.2\%) had attended high school level education. In addition half of the respondents $(50.5 \%)$ said they were housewives. 
The religion followed for $91.8 \%(180)$ was Orthodox Christianity and the commonest household monthly income reported by $55.6 \%(109)$ pregnant mothers ranged from 5001500 Birr. Almost all, i.e. 99.5\% (195 mothers) were not substance abusers, and unwanted pregnancies account $25.5 \%$. About $46.4 \%$ (91) were in the third trimester and the remaining $42.3 \%$ ( 83), $11.2 \%(22)$ were in the second and first trimesters respectively. In addition, 32\%(62) said they had delivered twice and $32.1 \%$ (63) said it third their third gestation. From the total of 196 respondents, 93.4\%(170) had no previous history of obstetric complication. Similarly, 97.4\%(191) were negative for sexually transmitted diseases.

Magnitude of antenatal depression: A total of 61 mothers scored 14 and above on BDI. Thus, the magnitude of antenatal depression was $31.1 \%$. Of these, $19.8 \%$ had mild depression, $8.2 \%$ had moderate depression while $3.1 \%$ had severe depression.

Half of the mothers (50\%) who came for ANC whose ages were in the age group of 15-20 years had depression. Of them, $12.5 \%$ had severe depression, and the remaining $87.5 \%$ was shared by mild and moderate depression equally (Figure $1)$.

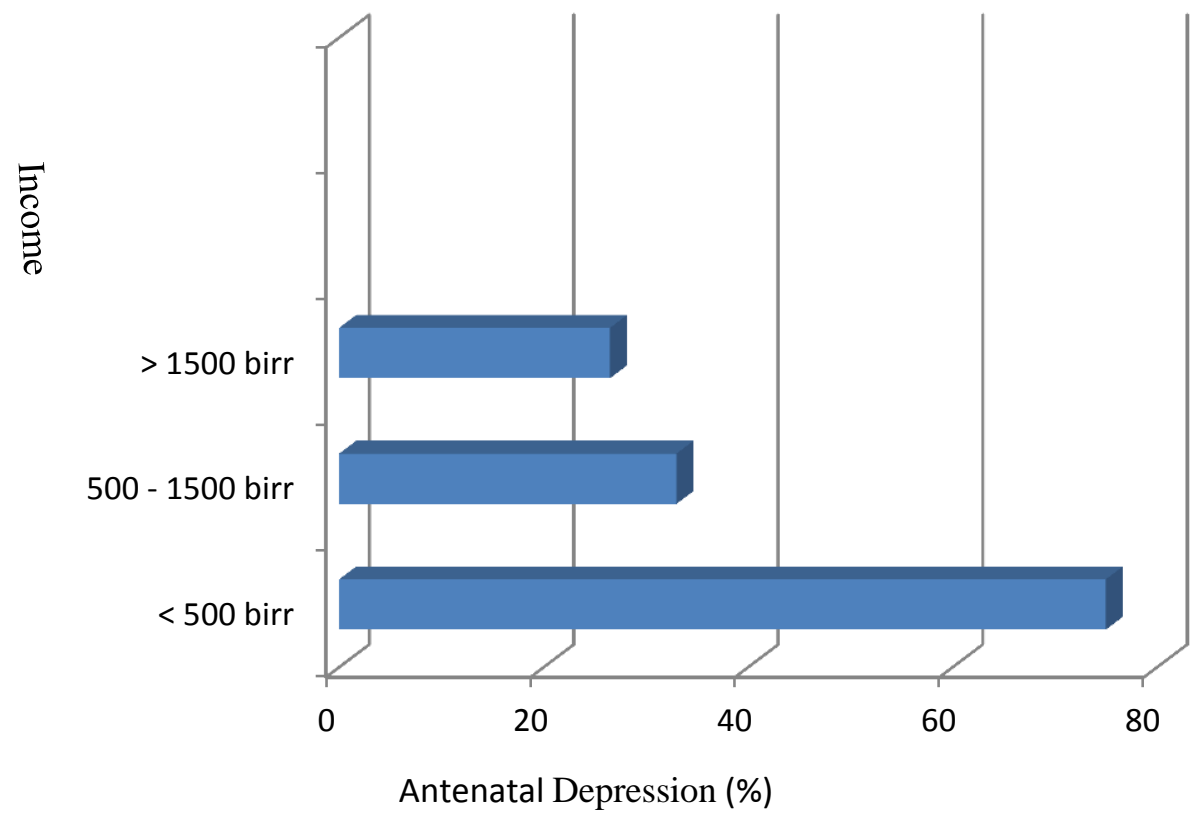

Figure 1: Magnitude of antenatal depression across the age group of pregnant women, Maichew, Ethiopia, June 2015

Antenatal depression among married pregnant mothers was found to be $28.2 \%$, and among these mothers $7.8 \%$ had severe depression. In addition, half of the mothers who are divorced were found to have depression. This $50 \%$ is equally shared by mild and moderate depression. Severe depression was absent among divorced mothers.

From the pregnant mothers who said that they never went to formal education, $42.9 \%$ had depression, and of them, 33.3\% had mild depression. Also, it was $30.9 \%$ among mothers who quit schooling from elementary level. Similarly, depression among pregnant mothers who were in college or higher level of education was $36 \%$. The magnitude of mild and moderate depression was $76.9 \%$ and $23.1 \%$ respectively. Severe depression was absent in mothers who were in college or higher level of education. Depression in mothers whose monthly household income was less than 500 Birr was $75 \%$, and $33 \%$ it was among those whose level of income is between 50 - 1500 Birr (Figure 2).

DOI: http://dx.doi.org/10.4314/ejhs.v27i1.8 


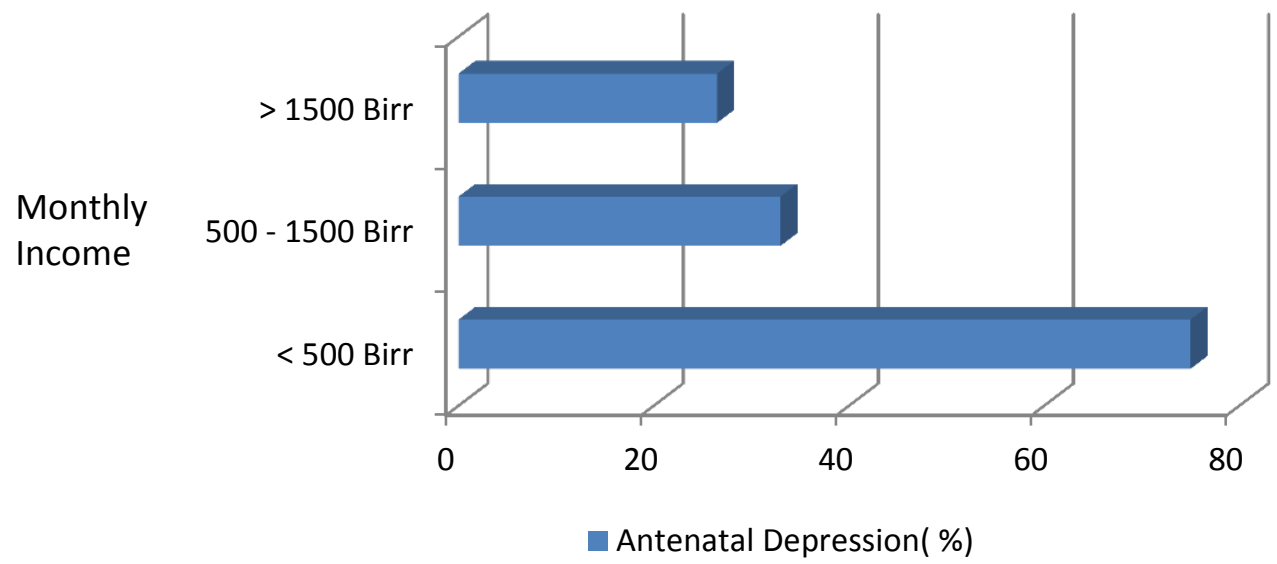

Figure 2: Magnitude of antenatal depression in relation to household monthly income, Maichew, Ethiopia, June 2015

The prevalence of depression in the first, second and third trimester of pregnancy was $36.4 \%$, $26.5 \%$ and $34.1 \%$ respectively (Table 1 ). Depression in mothers who had never given birth before (first pregnancy) accounts for $46.9 \%$; but it was $27 \%$ for women with second pregnancy.

Table 1: Magnitude of antenatal depression in relation to trimester of pregnancy among women at Maichew, Ethiopia, June 2015 ( $n=196)$.

\begin{tabular}{lll}
\hline Trimester & $\begin{array}{l}\text { Frequency } \\
(\%)\end{array}$ & $\begin{array}{l}\text { Antenatal } \\
\text { depression } \\
(\%)\end{array}$ \\
\hline First trimester & $22(11.2)$ & 36.4 \\
Second trimester & $83(42.3)$ & 26.5 \\
Third trimester & $91(46.4)$ & 34.1 \\
\hline
\end{tabular}

Depression in mothers who had history of obstetric complication accounted for $50 \%$. Of these, $16.7 \%$ had severe depression, $50 \%$ had moderate depression while $33.3 \%$ had mild depression (Figure 3).

The mutivariate analysis showed that women who were unmarried were about 4 times more likely to be depressed than married mothers
$(\mathrm{AOR}=4.07 \quad((95 \% \mathrm{CI} ; 1.18,14.04))$. Those pregnant women with low level of income (monthly income of $\leq 1500$ Birr) were 4 times more prone to develop depression than women who earn monthly income of more than 1500 Birr $((\mathrm{AOR}=4.06 ; 95 \% \mathrm{CI}(1.32,12.49))$. In addition, housewives, compared to women who were involved in private jobs, were 4 times more likely to be depressed $(\mathrm{AOR}=4.24(1.38,13.03))$ [Table 2].

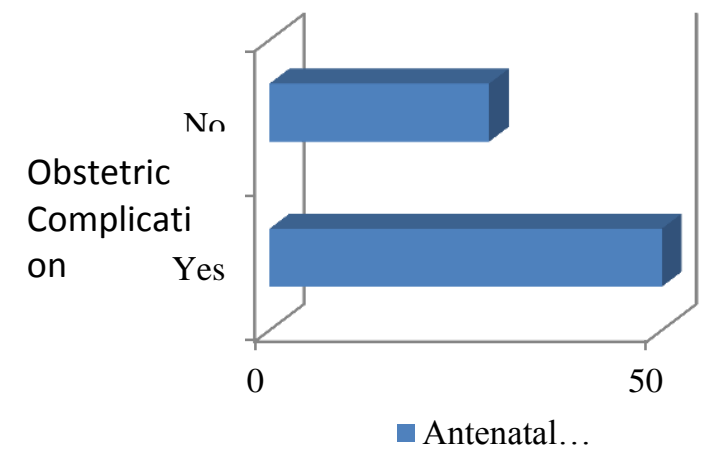

Figure 3: Magnitude of antenatal depression in relation to previous history of obstetric complication among pregnant women, Maichew, Ethiopia, June 2015 
Table 2: Association between antenatal depression and other factors, Maichew, Ethiopia, June 2015.

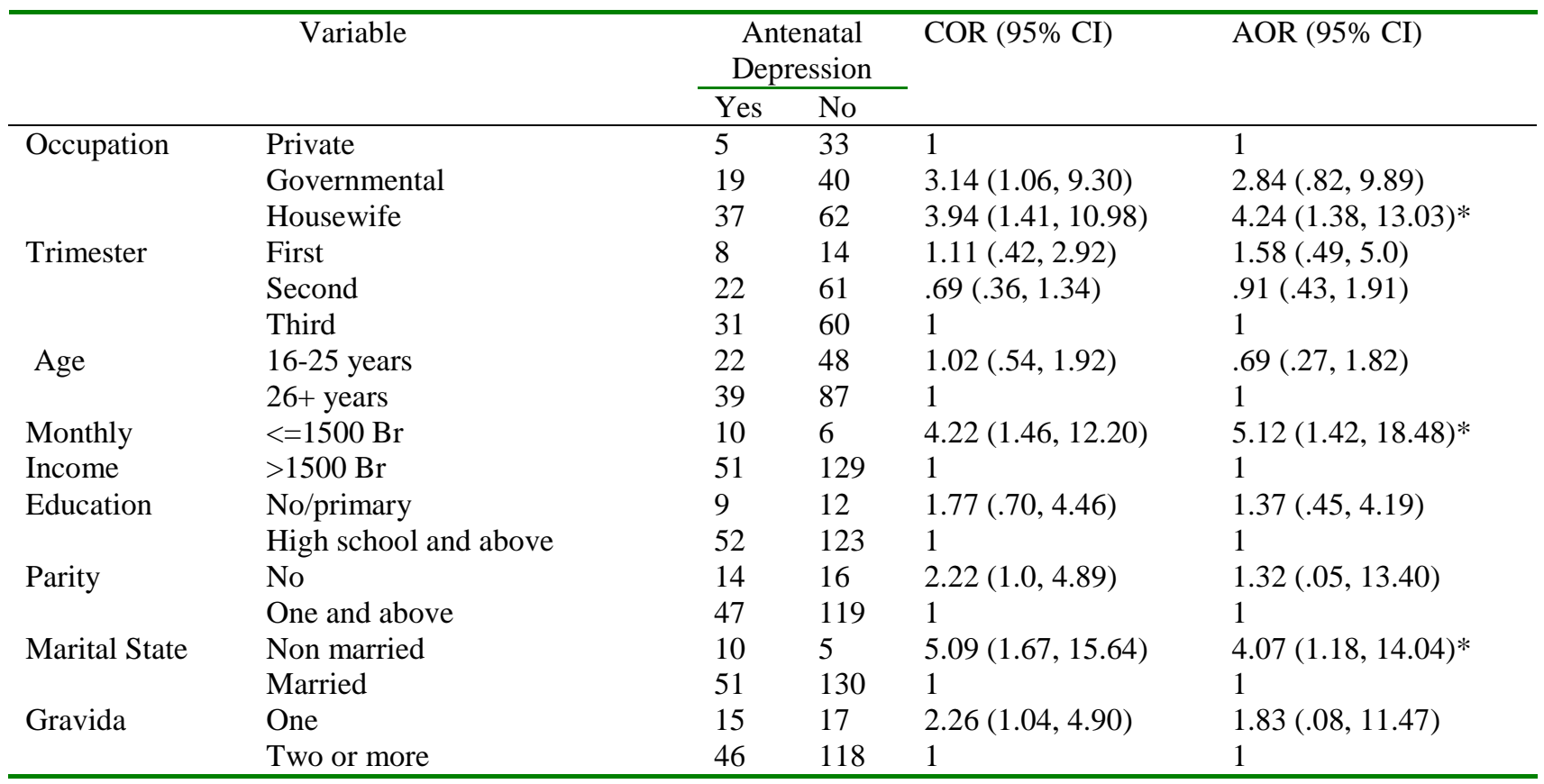

*significantly associated, $\mathrm{COR}=$ crude odds ratio, $\mathrm{AOR}=$ adjusted odds ratio, $\mathrm{CI}=$ confidence interval

\section{DISCUSSION}

This study attempted to determine the magnitude of antenatal depression and its socio-demographic variation among pregnant mothers at health institutions of Maichew Town. The magnitude of antenatal depression was $31.1 \%$. Our findings show that the magnitude of antenatal depression was almost similar with Brazil (38\%), South Africa (20-47\%) $(29,30,37))$, developed countries (oscillates between 5-30\%) and Addis Ababa (25$30 \%)(22,23,31,32,33)$. Still, it is a little higher than studies from Gondar (23\%) and Shashemene $(25.6 \%)(34,35)$. This might be due to larger sample size in both studies compared to the current study. Studies in Japanese (5.6 \%, (38)), East Asia (4.8\% (39)), in Navi Mumbai $(9.18 \%$ (28)) showed lower magnitude than this study. The reason for this variation could be the mothers' ego strength and proper care for the pregnant mothers; there also are cultural variations.

Monthly household income of pregnant mothers was a significant variable that holds a large percentage in antenatal depression. Studies in developing countries (28), South Africa, i.e. outskirts of Cape Town (37) and Shashemene in
Ethiopia (35) showed that pregnant mothers with low income had more depression during pregnancy than those who have medium and high monthly household income. This can be due to worrying for parents' and the coming babies' basic needs for a better quality life.

Low level of income in our study is similarly related to depression during pregnancy. In addition, those pregnant women who are employed in private activities are less likely to develop depression than housewives. Some housewives have additional job in which they can engage in different settings besides their household activities whereas others do not have such opportunities due to either low socioeconomic status or huge burden in household activities. And, these issues may in turn contribute to emotional disturbance. This fact was similarly found at Gondar, Ethiopia (34).

In the current study, unmarried women were at risk of depression. It can be explained by poor social support during pregnancy that was common predictor of depression as indicated in prior studies $(32,34,35)$. This finding was similarly reported in earlier studies at South Africa, Addis Ababa and Shashemene $(29,30,33,35)$. 
Unwanted pregnancy is also another variable which has a great role on depression during pregnancy as reported in prior studies (22 and 28). However, it did not show a significant association in this study. Women with younger age were more likely to have antenatal depression compared to other age groups (37). However, there was no significant association between age group and antenatal depression in the current study.

Pregnant mothers with history of obstetric complication were more prone to antenatal depression as found in earlier studies. This may be due to fear of having another complication in the current pregnancy $(28,35)$. In this study, $25.5 \%$ of pregnancies were unwanted, but no significant association was observed between unwanted pregnancy and antenatal depression.

Moreover, antenatal depression was common during pregnancy that was more prevalent among housewives and in those with low level of income. Hence screening activities for depression in antenatal care service should be emphasized with particular attention to unmarried mothers, mothers with low level of income and mothers who are housewives.

We have summarized limitations of this study as follows. The study was facility based; so, it is not e representative of the community as the ANC rate is not $100 \%$ in the area. This is the major limitation. In addition, the sample size used was small which might hinder the assessment of different variables. The other point is concerns the research tool we employed; the assessment tool BDI is not validated in Tigrigna.

\section{ACKNOWLEDGEMENT}

We express our appreciation to College of Health sciences, Mekelle University. Public health facilities at Maichew, data collectors and study participants are also highly acknowledged for their valuable contributions to this work.

\section{REFERENCES}

1. Mental health aspects of women's reproductive health, a global review of literature. Geneva, World Health Organization/United Nations Population Fund, 2009.
2. Gentile $\mathrm{S}$. The safety of newer antidepressants in pregnancy and breast feeding. Drug Saf. 2005; 28:137- 52.

3. Bonari L, Pinto N, Ahn E, Einarson A, Steiner M, Koren G. Perinatal risks of untreated depression during pregnancy. Canadian Journal of Psychiatry. 2004; 49: 726-735.

4. Sharma V. Pregnancy and bipolar disorder: a systematic review. J Clin Psychiatry. 2012; 73: $1447-1455$.

5. Marzuk M,et al. Lower risk of suicide during pregnancy. Am J Psychiatry. 1997; 154: 122.

6. Appleby L. Suicide during pregnancy in the first postnatal year. BMJ. 1991; 302: 137-40.

7. Pearson K, Nona's R, Cohen LS. Practical guidelines for the treatment of Psychiatric disorders during pregnancy. 2002:115-23.

8. Orr S, Miller C. Maternal depressive symptoms and the risk of poor pregnancy outcome. Review of the literature and preliminary findings. Epedemiol Rev. 1995; 17: 165-71.

9. Murray L. The impact of postnatal depression on infant development. J Child Psychol Psychiatry. 1997; 77: 99-101.

10. Weinberg M, Tronick E. The impact of maternal psychiatric illness on infant development. J Clin Psychiatry. 1998; 59: 53.

11. Chang HY, Keyes KM, Lee KS, Choi A, Kim SJ, Kim KW, et al. Prenatal maternal depression is associated with low birth weight through shorter gestational age in term infants in Korea. Early Hum Dev. 2014; 90: 15-20.

12. Marcus S. Depression during pregnancy: rates, risks and consequences. Mother risk Update. 2009; 16: $15-22$.

13. Austin M. Maternal stress and obstetric and infant outcomes: epidemiological findings and neuro endocrine mechanisms. Aust $N$ Z J Obstet Gynaecol. 2000; 40: 331-337.

14. Glover V. Maternal stress or anxiety in pregnancy and emotional development of the child. $\mathrm{Br} J$ Psychiatry. 1997; 171: 105-106.

15. Nusrat H, Tariq M, Farhat J, Meher H, Asia P, Qamar S, et al. Antenatal depression is not associated with low-birth weight: a study from urban Pakistan. Affective disorders and Psycho somatic research. Frontiers in Psychiatry. December 2014. http://dx.doi.org/10.3389/fpsyt.2014.00175

16. Heron J, O'Conner TG, Evans J, Golding J, Glover V; ALSPAC Study Team. The course of anxiety and depression through pregnancy and the postpartum in a community sample. $J$ Affect Disord. 2004; 80: 65-73.

DOI: http://dx.doi.org/10.4314/ejhs.v27i1.8 
17. Da Costa D, Larouche J, Dritsa M, Brender W. Psychosocial correlates of pre partum and postpartum depressed mood. J Affect Disord. 2000; 59: 31-40.

18. Pajulo M, Savonlathi E, Sourander A, Helenius H and Piha J. Antenatal depression, substance dependency and social support. J Affect Disord. 2001; 65: 9-17.

19. Misri, S, Kendrick K, Oberlander TF, et al "Antenatal Depression and Anxiety Affect Postpartum Parenting Stress: a Longitudinal Study. Can J Psychiatry. April 2010; 55 (4): 22-8.

20. Esra Yazici, Tulay Sati Kirkan,Puren Akcali Aslan,Nazan Aydin, and Ahmet Bulent Yazici. Untreated depression in the first trimester of pregnancy leads to postpartum depression: high rates from a natural follow-up study. Neuropsychiatr Dis Treat. 2015; 11:405-411.

21. Susan Pawlby, Dale Hay, Deborah Sharp, Cerith S. Waters, Carmine M. Pariante. Antenatal depression and offspring psychopathology: the influence of childhood maltreatment. The British Journal of Psychiatry Jul 2011; 199 (2): 106-112.

22. Shaunak Ajinkya. Pradeep RJ and Nimisha NS. Risk factors predispose to depression during pregnancy. Ind Psychiatry J. 2013; 22(1): 37-40

23. Snaith P. What do depression rating scales measure? British Journal of Psychiatry. 1993; 163: 293-298.

24. Qiu J. Reproductive-related depression in women: a review of two decades of research in China. AsiaPac Psychiatry. 2010; 2:19.

25. Shi S. An investigation of the prevalence of anxiety or depression and related risk factors in Chinese women during pregnancy and postpartum. BMC. 2007; 21: 254-258

26. Lee A. Prevalence, course, and risk factors for antenatal anxiety and depression. Obstet Gynecol. 2007; 110: 1102-1112.

27. Hsu C. The prevalence and predictors of prenatal depression. J Evid Based Nurs. 2008; 4: 149-156.

28. Shaunak A, Pradeep R, Jahav and Nimisha N. Sirvastava. Depression during Pregnancy: Prevalence and Obstetric risk factors among pregnant women attending a tertiary care hospital in Navi Mumbai. Industrial Psychiatry Journal. 2013; 22(1): 37-40.

29. Tamsen Jean Rochat, Mark Tomlinson, Till Bärnighausen, Marie-Louise Newell and Alan Stein. The prevalence and clinical presentation of antenatal depression in rural South Africa. Journal of Affective Disorders. 2011; 135 (1): 362-373

30. Kirsty Brittain, Landon Myer, Nastassja Koen, Sheri Koopowitz, Kirsten A D, Whitney Barnet, et al. Risk Factors for Antenatal Depression and Associations with Infant Birth Outcomes: Results from a South African Birth Cohort Study. Paediatric and Perinatal Epidemiology. November 2015; 29(6): 505-514. doi: 10.1111/ppe.12216

31. Abera Biratu and Demewoz Haile. Prevalence of antenatal depression and associated factors among pregnant women in Addis Ababa, Ethiopia: a cross-sectional study. Reprod Health. 2015; 12: 99. doi: 10.1186/s12978-015-0092-x

31. Mamo D and Worku A. Prevalence and Associated factors of Mental Distress during Pregnancy among Antenatal Care attendants at Saint Paul's Hospital, Addis Ababa. Addis Ababa University electronic library. Available at: www.comtecmed.

33. Asmeret Andebirhan. A Screening for Antenatal Depression: a Formative Study for Development of a Perinatal Mental Health Liaison Service in Zewditu Hospital. Addis Ababa University electronic library. Available at: etd.aau.edu.et/bistream/123456789/1/Asmeret\%20 Andebirhan

34. Tadesse Awoke Ayele, Telake Azale, Kassahun Alemu, Zewditu Abdissa, Haregewoin Mulat, and Abel Fekadu. Prevalence and Associated Factors of Antenatal Depression among Women Attending Antenatal Care Service at Gondar University Hospital, Northwest Ethiopia. PLOS One. 2016; 11(5): e0155125. doi: 10.1371/journal.pone.0155125

35. Assefa Gemta W. Prevalence and factors associated with antenatal depression among women following antenatal care at Shashemane health facilities, South Ethiopia. Annals of Global Health. January-February, 2015; 81 (1): 90

36. Central Statistics Agency, Ethiopia. Ethiopian Demographic and Health Survey. Tigray Region. EDHS. 2011

37. Mary Hartley, Mark Tomlinson and Mary Janelrotheram-Borus. Depressed mood in pregnancy: Prevalence and correlates in two Cape Town peri-urban settlements. BMC Reproductive Health. 2011; 8:9

38. Kitamura T, Yoshida K, Okano T, et al. Multi centre prospective study of perinatal depression in Japan: Incidence and correlates of antenatal depression and postnatal depression. Arch Womens Ment Health. 2006; 9:121-130.

39. Qiao YX, Wang J, LiJ and Ablat A. The prevalence and related risk factors of anxiety and depression symptoms among Chinese pregnant women in Shanghai. Aust N Z Obstet Gynaecol. 2009; 49 (2): 185-190. 